\title{
Revision
}

\section{LECTINAS CON DOMINIO DE LEGUMINOSA: CARACTERÍSTICAS ESTRUCTURALES Y UTILIDAD COMO AGENTES INSECTISTÁTICOS E INSECTICIDAS}

\author{
Review \\ LEGUME LECTINS DOMAIN: STRUCTURAL CHARACTERISTICS AND \\ INSECTISTATIC AND INSECTICIDAL ACTIVITIES \\ Zulma Yanira Casas Corredor ${ }^{1,2}$, Edgar Antonio Reyes Montaño ${ }^{2 *}$, y Nohora Angélica Vega Castro ${ }^{2}$ \\ ${ }^{1}$ Departamento de Nutrición y Bioquímica, Pontificia Universidad Javeriana, KR 7 \# 40-62, Bogotá, \\ Colombia. \\ ${ }^{2}$ Departamento de Química, Universidad Nacional de Colombia, Avenida Carrera 30 \# 45, Bogotá, \\ Colombia. \\ *Autor para correspondencia E-mail: eareyesm@unal.edu.co
}

\section{RESUMEN}

Esta revisión busca identificar las características de las lectinas vegetales con dominio de leguminosa que determinan su actividad insectistática e insecticida. Se realizó una búsqueda sistemática de literatura en las bases de datos Medline, Science Direct (Elsevier), Pubmed, Lilacs, SCOPUS, Web of Science (ISI) con el uso de palabras claves relevantes. La información recolectada fue clasificada y seleccionada en términos de su calidad y relevancia. La revisión tomó como base la información de las lectinas de Phaseolus vulgaris (PHA), Glechoma hederacea (Gleheda), Canavalia ensiformis (Concanavalina A), Griffonia simplicifolia (GSII) y Pisum sativum (PSA) y lectinas de leguminosas estudiadas en Colombia por el Grupo de Investigación en Proteínas. Se evidenció que las lectinas vegetales con dominio de leguminosa poseen rasgos estructurales caracterizados por un alto porcentaje de láminas beta asociados en estructuras diméricas o tetraméricas y que presentan varios sitios de reconocimiento a azúcares específicos, entre los cuales se destaca la manosa. Adicional a estas características, estas lectinas pueden interactuar con el sistema digestivo de insectos plaga y generar una disminución en su capacidad de absorción intestinal. Como conclusión, se puede señalar que es insuficiente la información sobre las señales que se activan y los efectos deletéreos de las lectinas con dominio de leguminosa sobre los insectos.

Palabras clave: insectistático, insecticida, leguminosa, lectina.

\section{ABSTRACT}

This review aims to identify the characteristics of legume lectins that determine their insecticidal and insectistatic activities. A systematic review of the literature was conducted in Medline, Science Direct (Elsevier), Pubmed, SCOPUS, Web of Science (ISI) and Lilacs databases by using relevant keywords. The information collected was classified and selected in terms of quality and relevance. This review is mainly based on information of Phaseolus vulgaris (PHA), Glechoma hederacea (Gleheda), Canavalia ensiformis (ConA), Griffonia simplicifolia (GSII) and Pisum sativum (PSA) lectins and other legume lectins studied by the Protein Research Group in Colombia. It was evidenced that legume lectins have structural features characterized by a high percentage of beta sheet structures that form dimeric or tetrameric assemblies and present several specific sugars recognition sites, 
including mannose. In addition, these lectins can interact with the digestive system of insect pests and produce a decrease in intestinal absorption capacity. As conclusions, there is lack of knowledge about signals that are activated and the deleterious effects of legume lectins domain on insects.

Key words: insectistatic, insecticide, legume, lectin.

\section{INTRODUCCIÓN}

Las plantas emplean sistemas de defensa sofisticados contra un gran número de insectos y patógenos, que presentan diferentes estilos de vida y estrategias de infección (Jones y Dangl, 2006; Chen, 2008). Evolutivamente las plantas han desarrollado mecanismos de defensa constitutivas e inducibles (Chen, 2008). Las defensas constitutivas son el resultado de la generación de barreras físicas y/o químicas que existen antes del ataque del insecto (Jones y Dangl, 2006), por ejemplo, estas defensas pueden repeler a los insectos fitófagos a través de una toxicidad directa o mediante la reducción de la digestibilidad de los tejidos de las plantas (Jones y Dangl, 2006). Por el contrario, las defensas inducibles se activan como respuesta a daños tisulares inducidos por el ataque de los insectos (Jones y Dangl, 2006; Chen, 2008). Estas estrategias son capaces de prevenir el ataque de la mayor parte de los insectos herbívoros, aunque se ha encontrado que un reducido número de insectos son capaces de adaptarse a la defensa específica de las plantas (Mello y Silva-Filho, 2002).

Las defensas constitutivas pueden ser clasificadas como anti-nutricionales y tóxicas (Chen, 2008). Las defensas anti-nutricionales en la pre-digestión limitan el suministro de alimentos (Chen et al., 2007), lo que conlleva a una reducción en la ingestión y en el valor nutricional de los mismos, ya sea por la eliminación de nutrientes esenciales y/o la inhibición de la ingestión (Chen, 2008). En las defensas tóxicas, las plantas producen péptidos y proteínas como moléculas de defensa contra el ataque de insectos fitófagos (Tanji et al., 2006), lo que induce daños físicos y alteraciones químicas en el insecto (Chen et al., 2007).

Las lectinas vegetales son proteínas de gran estabilidad (Chen et al., 2007; Chen, 2008) que tienen la capacidad de unirse reversiblemente con alta afinidad y especificidad a diferentes tipos de carbohidratos (Sharon, 2007) y que son sintetizadas durante el desarrollo de las semillas, la germinación y el crecimiento de las plantas, y que pueden ser abundantes en órganos de las plantas, tales como raíces, hojas y tallos (Wang et al., 2003). Las lectinas de origen vegetal al ser ingeridas (Vasconcelos y Oliveira, 2004; Vandenborre et al., 2011; Al Atalah et al., 2014) interaccionan con proteínas glicosiladas de las células del intestino (Vasconcelos y Oliveira, 2004; Michiels et al., 2010) lo que genera efectos anti-nutricionales (Sauvion et al., 2004a; Upadhyay y Singh, 2012), reproductivos (Sadeghi et al., 2006) y tóxicos en las larvas de los insectos que los consumen (Melander et al., 2003; Lehrman et al., 2007a; Delatorre et al., 2007; Al Atalah et al., 2014). Es interesante mencionar que estas proteínas son inocuas en humanos y en algunos larvas de insectos benéficos (Vasconcelos y Oliveira, 2004; Lehrman, 2007b; Vandenborre et al., 2011).

La familia mejor definida y con más potencial insectistático e insecticida de este grupo de proteínas son las lectinas con dominio de leguminosas. Por ejemplo, se ha demostrado la actividad de las lectinas de Canavalia ensiformis (Concanavalina A (ConA)), Pisum satioum (PSA) en el crecimiento y supervivencia de las larvas del escarabajo de polen Meligethes aeneus (Melander et al., 2003) y de Glechoma hederacea (Gleheda) para escarabajo de la patata Leptinotarsa decemlineata (Wang et al., 2003). En el presente artículo, inicialmente se hace una revisión sistemática de las características estructurales de las lectinas con dominio de leguminosas Phaseolus vulgaris (PHA), Glechoma hederacea (Gleheda), Canavalia ensiformis (ConA), Griffonia simplicifolia (GSII) y Pisum sativum (PSA) y posteriormente se relacionan con sus mecanismos de acción como agentes insectistático e insecticidas.

\section{Definición, función y clasificación de lectinas de plantas}

Las lectinas son proteínas de unión a carbohidratos de origen no inmune, y que poseen por lo menos un dominio no catalítico que se une de forma reversible a mono u oligosacáridos específicos (Sharon, 2007). Las lectinas están ampliamente distribuidas en la naturaleza, por ejemplo se encuentran en virus (Sharon y Lis, 2004; Van Breedam et al., 2013), bacterias (Sharon y Lis, 2004; Perret et al., 2005; Topin et al., 2013), hongos (Bovi et al., 2011; Wang et al., 2012; Gao et al., 2013; Hamshou et al., 2013), animales (Wei et al., 2012; Zhu et al., 2013) y plantas (Singh et al., 2006; Arcoverde et al., 2014).

Estudios previos han encontrado que las lectinas vegetales están involucradas en el almacenamiento y transporte de los hidratos de carbono en las semillas, en el crecimiento de la planta, en la unión de bacterias fijadoras de nitrógeno a la raíz, la inhibición del crecimiento de hongos y en 
los mecanismos de defensa contra insectos fitófagos (Sanz-Aparicio et al., 1997; Vandenborre et al., 2011). Adicionalmente, se ha encontrado que pueden mediar varios procesos biológicos, entre los que se encuentran: el reconocimiento célula-célula (interacción huésped-patógeno) (Sharon y Lis, 2004; Naeem et al., 2007) y la división celular (estimulación mitogénica de linfocitos) (Wecksler et al., 1968). A su vez, han sido utilizados para el reconocimiento de receptores de membrana y detección de algunos tumores (Sharon y Lis, 2004). Se ha sugerido que esta diversidad de funciones está mediada por las diferencias en las uniones a carbohidratos de algunas lectinas (Dam et al., 2005).

Inicialmente las lectinas se clasificaron con base en su habilidad para reconocer y unir azúcares específicos (Goldstein et al., 1997). Acorde con estos parámetros, las lectinas se subdividieron en cinco grupos, dependiendo de la afinidad a monosacáridos tales como: D-manosa/D-glucosa, D-galactosa/N-acetil-D-galactosamina, $\mathrm{N}$-acetil-D-glucosamina, L-fucosa y ácido N-acetilmuramico (Goldstein et al., 1997). Sin embargo, Van Damme et al. (1998b) han postulado un nuevo sistema de clasificación de las lectinas de plantas teniendo en cuenta la arquitectura de los dominios de unión a carbohidratos. Esto debido, a que algunas lectinas son promiscuas y pueden unir más de un tipo de carbohidrato. En este sistema, las lectinas han sido clasificadas en merolectinas, hololectinas, chimerolectinas y superlectinas. Las merolectinas son lectinas que tienen un solo sitio de unión a carbohidratos; por su carácter monovalente este grupo de lectinas no puede aglutinar células. Las hololectinas comprenden todas las lectinas que tienen dos o más sitios idénticos o con una alta homología en el reconocimiento de carbohidratos, lo que les permite aglutinar células y/o formar precipitados de glicoconjugados. Las chimerolectinas, a diferencia de las dos anteriores, contienen uno o más dominios de unión a carbohidratos y adicionalmente tienen un dominio que tiene actividad catalítica o biológica. Finalmente, las superlectinas poseen al menos dos dominios de unión a carbohidratos, pero difieren de las hololectinas debido a que sus sitios de unión son capaces de reconocer azúcares no relacionados estructuralmente.

Estudios recientes han llevado a una clasificación más compleja en la que se tiene en cuenta la estructura terciaria y las relaciones evolutivas existentes, ya que se ha encontrado que las lectinas poseen múltiples dominios embebidos en arquitecturas complejas (Van Damme et al., 2008). Esta clasificación ha dado origen a doce familias: aglutininas con dominio Agaricus bisporus, amaranthins y homólogos de la clase V Quitina; dominio cyanovirina; aglutininas con dominio Euonymus europaeus; aglutinina con Dominio $\mathrm{Ga}$ lanthus nivalis; Dominio heveina; jacalins; lectina con Dominio Leguminosa; Dominio Lys; aglutinina con Dominio Nicotiana tabacum; Dominio Ricin-B (Van Damme et al., 2008).

A pesar que la gran mayoría de lectinas tienen efectos tóxicos sobre diferentes insectos fitófagos, se ha observado que el tipo de domino que posean juega un rol importante en su actividad biológica. Por ejemplo, aquellas que tienen similitud estructural de su dominio de lectina con la concavalina A y preferencia a interactuar con manosas, tienen el mayor potencial como agentes insectistáticos e insecticidas. Tal es el caso de las lectinas con dominio leguminosa, que se han encontrado afectan la ovoposición, crecimiento, pupación y sobrevivencia de diferentes insectos plaga (Zhu et al., 1999; Machuka et al., 2000; Melander et al., 2003; Sadeghi et al., 2006 ).

\section{Estructura de lectinas con dominio de leguminosas}

Se ha reportado que las lectinas de leguminosas por lo general se componen de dos o cuatro subunidades, con un peso molecular aproximado de 25-30 kDa por subunidad. Cada subunidad tienen un sitio de unión a carbohidratos (Sharon y Lis, 1990; Abhilash et al., 2013). Estudios realizados (Banerjee et al., 1996; Srinivas et al., 2001)) reportaron que cada subunidad de la proteína tiene la forma de un domo constituido por tres hojas $\beta$; una trasera, una delantera y una más pequeña con cinco bandas cuya función es mantener a las dos anteriores unidas. La hoja $\beta$ trasera se caracteriza por estar constituida por seis laminas anti-paralelas planas (Fig. 1a hojas oscuras), una hoja curva de siete laminas antiparalelas $\beta$ (Fig. 1a hojas grises), y una hoja superior de cinco hebras unidas por lazos de diferentes longitudes (Fig. 1a más claras). Los monómeros de lectinas esencialmente varían en la longitud y forma de sus "loops" (Banerjee et al., 1996; Srinivas et al., 2001). A este plegamiento típico lo designaron como el "plegamiento de lectina de leguminosa" (Fig. 1a) que también se ha encontrado en otras proteínas (Loris et al., 1998; Vijayan y Chandra, 1999; Srinivas et al.,2001) que se caracteriza por la presencia de iones metálicos como parte esencial de la estructura nativa, de la actividad biológica de la proteína (Novakova et al., 1981; Abhilash et al., 2013) y del mantenimiento de una conformación que permita la correcta disposición de los sitios de unión a carbohidratos (Pusztai et al., 1990; Abhilash et al., 2013). Adicionalmente, se ha encontrado que la unión de estos iones está mediada por aminoácidos como el ácido aspártico y la asparagina que están conservados en las 
diferentes lectinas de leguminosas (Deacon et al., 1997; Rao et al., 1998; Sharon y Lis, 2001; Abhilash et al., 2013).

En la Fig. 1a se ilustra la estructura terciaria de ConA, y en la Fig. 1b la forma como se unen dos monómeros por sus hojas de seis laminas $\beta$ dando lugar a la formación de doce hojas unidas. (Srinivas et al., 2001).

El análisis de la estructura de diferentes monómeros de lectinas con dominio de leguminosas han mostrado una alta similitud estructural (Chandra et al., 2001; Bezerra et al., 2013). Por ejemplo, la comparación de secuencias disponibles en bases de datos de las lectinas dominio de leguminosas, muestran una similitud en su estructura primaria de alrededor del $20 \%$ en aminoácidos idénticos y un $40 \%$ en aminoácidos similares (consulta realizada en bases de datos GenBank y Protein Data Bank en octubre 20 del 2013). Los aminoácidos conservados en las lectinas incluyen aquellos que corresponden al "sitio de unión" del carbohidrato y los que coordinan la unión de iones metálicos.

En la Concavanalina A, el sitio de unión a metales está situado en el lado amino terminal de la cadena de polipéptidos (Fig. 2), cada subunidad de la lectina tiene un ácido aspártico en posición 10 y 19, una asparagina en posición 14, una histidina en posición 24, una serina en posición 34, un ácido glutámico en posición 8 , y una tirosina en posición 12; estos aminoácidos están implicados en la unión a iones divalentes como el calcio y manganeso (Permyakov, 2009).

También se ha reportado que las lectinas de a.

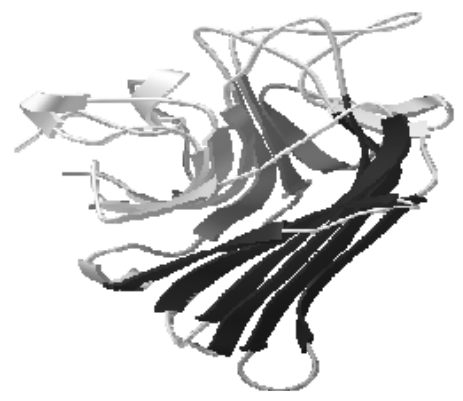

b.

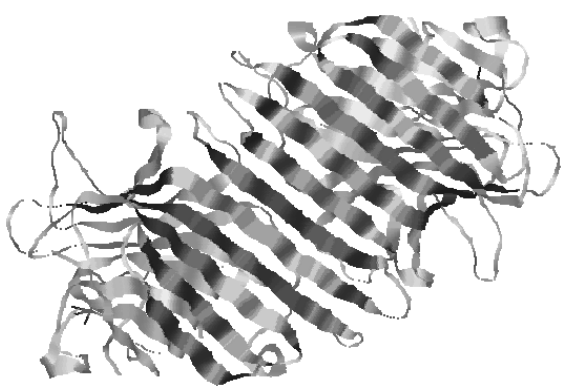

Fig. 1. Estructura terciaria de lectinas de leguminosas. (a) Ejemplo de ConA, el monómero mejor descrito con plegamiento conocido como "Jelly roll"; (b) Dimerización de la ConA.

Fuente: Srinivas et al., 2001.

Fig. 1. Tertiary structure of legume lectins. (a) ConA is the best described example of folding monomer known as "Jelly roll"; (b) Dimerization of ConA.

Source: Srinivas et al., 2001.

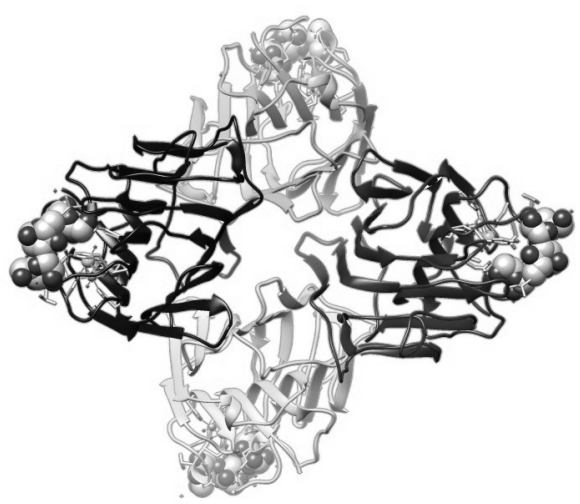

Fig. 2. Estructura tetramérica de Con $A$ obtenida por difracción de rayos $X$ a 2,35 Å (1CVN). El ligando trimanósido se indica con las esferas, así como el $\mathrm{Ca}^{2+}$ y el $\mathrm{Mn}^{2+}$. Adaptado de Varki et al., 1999.

Fig. 2. ConA tetrameric structure obtained by X-ray diffraction of $2.35 \AA$ (1CVN). Trimannoside ligand is indicated by the gray spheres as well as the $\mathrm{Ca}^{2+}$ and $\mathrm{Mn}^{2+}$ ions. Adapted from Varki et al., 1999. 
soja (Glicine max), guisantes (Pisum sativum), habas (Vicia faba), y lentejas (Lens esculenta), tienen aminoácidos conservados que están implicados en la unión de metales. La excepción es el residuo de tirosina en la posición 12 de ConA, que se sustituye por fenilalanina en las otras lectinas de leguminosas (Sharon y Lis, 2001).

Una propiedad importante de las lectinas es su sitio de unión específico a carbohidratos, como se ilustra en la Fig 3. En este sitio de reconocimiento las interacciones entre las lectinas y los carbohidratos se hacen principalmente a través de fuerzas de Van der Waals (Loris et al., 1998; Loris, 2002). A su vez, se ha establecido que la estabilidad de la estructura nativa de la mayoría de las lectinas es dependiente de las interacciones hidrofóbicas (Loris et al., 1998; Chandra et al., 2001; Abhilash et al., 2013). Diferentes estudios han demostrado que las lectinas se caracterizan por tener cavidades hidrofóbicas en su estructura de importancia para su función (Abhilash et al., 2013; Chandra et al., 2001). Algunas lectinas están compuestas de subunidades con diferentes sitios de unión. Estos incluyen la lectina de frijol rojo, Phaseolus vulgaris, la cual se compone de dos subunidades diferentes que se combinan en cinco formas tetrámericas disímiles unidas no covalentemente. Las subunidades tienen diversas especificidades por los receptores de la superficie celular, cada combinación se considera que tiene una función diferente. La especificidad de los sitios de unión de las lectinas sugiere que existen receptores endógenos de sacáridos en los tejidos de los que se derivan o en otras células o glicoconjugados con los que la lectina está especializada para interactuar (Sharon y Lis, 2001).

Generalmente las lectinas se consideran glicoproteínas; sin embargo, la ConA no contiene carbohidratos unidos covalentemente por lo que se ha sugerido que se sintetizan como precursores glicosilados, como en el caso de la pro-ConA, glicoproteína inactiva, que durante el procesamiento post-traduccional se elimina la cadena lateral glicosilada (Van Damme et al., 1998a).

Además de las similitudes en estructura, las lectinas presentan diferencias en la forma y modos de organización de los monómeros, dímeros y tetrámeros. Pequeñas diferencias en las secuencias de aminoácidos en los monómeros, y la presencia o ausencia de glicosilación parece afectar su asociación monomérica, resultando en diferentes modos de interacción (Srinivas et al., 2001).

En las semillas del frijol común $P$. vulgaris, la fracción de proteína que tiene la propiedad de unir azúcares y capacidad hemoaglutinante (Goldstein y Hayes, 1978; Kaneda et al., 2002) se denomina fitohemaglutinina (PHA); la cual se compone de diferentes tetrámeros formados por dos cadenas de polipéptidos (PHA-E y PHA-L) que tienen propiedades de eritoaglutinación y leucoaglutinación, respectivamente (Hamelryck et al., 1996). Además, reconocen residuos terminales de galactosa y glicanos complejos, y son codificadas por dos genes, dlec1 y dlec2, respectivamente (Hoffman y Donaldson, 1985; Goossens et al., 1999). PHA-L y PHA-E tienen similar composición en carbohidratos y aminoácidos, pero diferente unión específica por complejos tipo $\mathrm{N}$-glicanos. Varios estudios correlacionan la di-

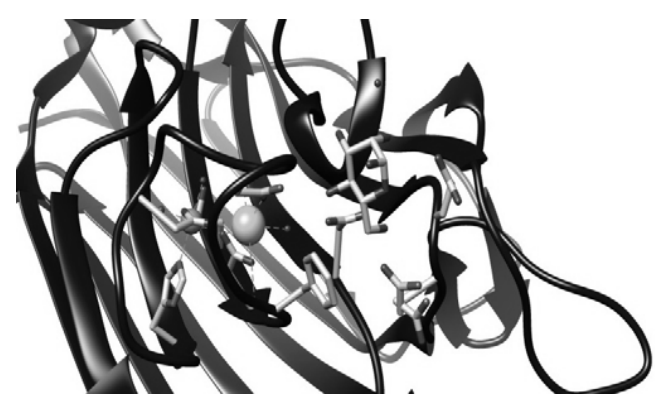

Fig 3. Vista detallada del sitio de unión de lectina con dominio de leguminosa (ilustrada para lectina $P$. angolensis). Se destaca un núcleo conservado de residuos de aminoácidos incluido un ion calcio (esfera gris) que está rodeado por dos bucles variables.

Adaptado de Loris, 2002.

Fig. 3. Detailed view of the binding site of legume lectin domain (illustrated for P. angolensis lectin). The core conserved amino acid residues, including a calcium ion (gray area) surrounded by two variable loops.

Adapted from Loris, 2002. 
ferente especificidad de las dos isolectinas con la diferencia en la secuencia de los aminoácidos del loop $\beta$ (Kaneda et al., 2002)

La tetramerización de lectinas con domiminio de leguminosas está estrechamente relacionada con su afinidad por oligosacáridos complejos. Las diferencias en las propiedades de unión a carbohidratos de algunas lectinas en reconocimiento celular y procesos de transducción de señales son reflejadas en sus propiedades biológicas (Dam et al., 2005)

Las lectinas se han purificado a partir de diferentes plantas. El contenido de lectina en algunas partes de las plantas son más altos, por ejemplo, 390 y $75 \mathrm{mg}$ de lectina purificada se recuperaron de $100 \mathrm{mg}$ de tubérculos de Remusatia vivípara (Bhat et al., 2010) y de raíces de Astrágalo mongholicus (Yan et al., 2005), respectivamente. El contenido de lectina en plantas no leguminosas es bajo, por ejemplo, Lam et al. (2009) obtuvieron $33 \mathrm{mg}$ de lectina a partir de $100 \mathrm{~g}$ de semillas de
Hibiscus mutabilis. Las lectinas o hemaglutininas se han purificado a partir de diferentes variedades de $P$. vulgaris y su concentración es baja en algunas variedades y altas en otras variedades, como se observa en la Tabla 1 (Aldana, 2000; Lam y Ng, 2011).

El Grupo de Investigación en Proteínas de la Universidad Nacional de Colombia ha realizado un estudio sistemático de la estructura y efectos biológicos de lectinas de la familia Fabaceae y Lamiaceae, Se ha detectado alta actividad de lectinas en cerca de 51 especies y se han aislado y caracterizado bioquímicamente las lectinas de Salvia bogotensis, Lepechinia bullata, Galactia lindenii, Dioclea lehmani, entre otras. Las tres primeras lectinas se han ensayado sobre cultivos de líneas celulares tumorales para reconocer antígenos tumorales o evaluar su actividad citotóxica (Aldana, J.C., 2000; Almanza et al., 2004; Vega y Pérez, 2006; Pérez et al., 2006; Pérez y Vega, 2007; Fernández-Alonso et al., 2009).

Tabla 1. Rendimientos de lectinas de plantas obtenidas mediante aislamiento cromatográfico de las semillas de diferentes cultivares de Phaseolus.

Table 1. Yields of plant lectins obtained by chromatographic isolation of seeds from different cultivars of Phaseolus.

\begin{tabular}{|c|c|c|c|c|}
\hline Cultivar phaseolus & $\begin{array}{l}\text { Purificación por } \\
\text { cromatografía }\end{array}$ & $\begin{array}{l}\text { Rendimiento } \\
\text { (mg/100g de } \\
\text { semilla) }\end{array}$ & $\begin{array}{l}\text { Especificidad } \\
\text { azúcar }\end{array}$ & Referencia \\
\hline Frijol anasazi & $\begin{array}{l}\text { Affi-gel azul, Mono } \\
\text { S y Superdex } 200\end{array}$ & 13 & No encontrada & $\begin{array}{l}\text { Sharma et al., } \\
2009\end{array}$ \\
\hline Frijol rojo oscuro & $\begin{array}{l}\text { DEAE-celulosa y } \\
\text { Affi-gel azul }\end{array}$ & 107 & No encontrada & Xia y Ng, 2006 \\
\hline Escumite bean & $\begin{array}{l}\text { Cromatografía } \\
\text { de afinidad } \\
\text { (membrana } \\
\text { glutaraldehido de } \\
\text { eritrocitos grupo } \\
\text { sanguíneo O) }\end{array}$ & $\begin{array}{l}163 \text { (total de } 4 \\
\text { isoformas) }\end{array}$ & $\begin{array}{l}\text { N-acetyllactosami- } \\
\text { ne-type glycans }\end{array}$ & $\begin{array}{l}\text { Castillo-Villa- } \\
\text { nueva et al., } \\
2007\end{array}$ \\
\hline $\begin{array}{l}\text { Extralong autumn } \\
\text { purple bean }\end{array}$ & $\begin{array}{l}\text { Azul-Sepharose, } \\
\text { Q-Sepharose, Mono } \\
\text { Q and Superdex } 75\end{array}$ & 35 & Galactosa & Fang et al., 2010 \\
\hline French bean 12 & $\begin{array}{l}\text { SP-Sepharose, } \\
\text { Affi-gel azul, } \\
\text { Q-Sepharose, y } \\
\text { Superdex } 200\end{array}$ & 4.8 & No encontrada & $\begin{array}{l}\text { Leung et al., } \\
2008\end{array}$ \\
\hline French bean 35 & $\begin{array}{l}\text { Azul-Sepharose, } \\
\text { Q-Sepharose and } \\
\text { Superdex } 75\end{array}$ & 1100 & No encontrada & Lam y $\mathrm{Ng}, 2010$ \\
\hline Frijol rojo & $\begin{array}{l}\text { Affi-gel azul y CM- } \\
\text { Sepharose }\end{array}$ & 27.5 & $\begin{array}{l}\text { Lactoferrina, ovalbu- } \\
\text { mina, triglobulina }\end{array}$ & Ye et al., 2001 \\
\hline
\end{tabular}

Adaptado de: Lam, S.K., y T.B. Ng. 2011. Appl Microbiology Biotechnol. 89(1):45-55 


\section{EFECTO INSECTISTÁTICO E INSECTICIDA DE LECTINAS CON DOMINIO DE LEGUMINOSAS}

Las lectinas con dominio de leguminosas tienen la capacidad de actuar como agentes insectistaticos e insecticidas, debido a la capacidad que tienen de unirse y/o reconocer carbohidratos de las células del intestino medio de los insectos. Esto se ha confirmado en diversos estudios donde se han alimentado insectos con dietas artificiales o con plantas transgénicas que expresan este tipo de lectinas (Vandenborre et al., 2011) y que inducen en el animal efectos insectistáticos, como pérdida de peso, disminución en la reproducción, e insecticidas caracterizados por la muerte de algunos de los insectos alimentados con esta lectina (Wang et al., 2003; Sauvion et al., 2004a).

\section{Efecto insectistático}

La mayoría de especies de plantas que se utilizan en protección vegetal exhiben un efecto insectistático más que insecticida, es decir, inhiben el desarrollo normal de los insectos. (Celis et., al 2008). De esta forma, se ha descrito que lectinas con dominio de leguminosas que han sido adicionadas a dietas artificiales o que se han expresado en plantas transgénicas tienen efectos insectistáticos, (Tabla 2) como disminución en el peso y tamaño de las larvas, así como también cambios en el color, retraso en el tiempo total de desarrollo y/o la fecundidad de insectos.

Uno de los insectos con más impacto económico en los que se ha evaluado el efecto insectistático de las lectinas con dominio de leguminosas es el del gorgojo del frijol (Callosobruchos maculatus), como se observa en la Tabla 2. Estudios en donde se han alimentado larvas de este insecto con lectinas aisladas de Sphenostylis stenocarpa (Machuka et al., 2000), de Phaseolus vulgaris (Gatehouse et al., 1984) y de Griffonia simplicifolia (GS-II) (Zhu et al., 1996) han mostrado que estas lectinas no solo inducen un retraso en el tiempo de desarrollo de las larvas sino también una disminución en su tamaño. Este efecto parece ser dosis dependiente, ya que dosis del $2 \%(\mathrm{p} / \mathrm{p})$ tiene una mayor efecto que dosis del 0,2\% (Machuka et al., 2000). Aunque el mecanismo por el cual estas lectinas afectan el tamaño y el desarrollo de las larvas no ha sido completamente dilucidado, se piensa que está mediado por la unión de las lectinas ingeridas a las células epiteliales del intestino, lo que afecta la absorción de nutrientes, la función secretora del intestino y el metabolismo (Gatehouse et al., 1984; Sauvion et al., 2004a; Van Damme, 2008; Michiels et al., 2010).

Adicionalmente se ha encontrado que cuando se suministra la lectina ConA como alimento a larvas del gusano del algodón (Helicoverpa armígera) en concentraciones de $1 \mathrm{mg} \mathrm{mL}^{-1}$ durante cinco días, se observa una disminución del 29\% en el peso de las larvas (Vasconcelos y Oliveira, 2004; Shukla et al., 2005) lo que sugiere que las lectinas de este tipo generan un efecto anti-nutricional que lleva a que la larva disminuya el consumo del alimento (Melander et al., 2003; Wang et al., 2003; Sauvion et al., 2004a). Este mismo efecto se ha encontrado en los estudios realizados por Melander et al. (2003) que utilizando la lectina PSA (aglutinina de Pisum satioum), y larvas del escarabajo del polen (Meligethes aeneus), encontraron que se redujo el peso de las larvas en un $79 \%$ cuando utilizó una solución al 1\% de la lectina (Melander et al., 2003).

Efectos más fuertes en el desarrollo han sido encontrados con la lectina Gleheda de la familia Lamiaceae (Glechoma hederacea) sobre las larvas del escarabajo de la patata (Leptinotarsa decemlineata) (Wang et al., 2003). En este estudio se encontró que larvas alimentadas con hojas de patata sumergidas en una solución de Gleheda no alcanzan el estado de pupa, lo que sugiere que esta

Tabla 2. Lectinas con dominio de leguminosas con actividad insectistática e insecticida. Table 2. Legume lectins domain with insectistatic and insecticidal activity.

\begin{tabular}{llll}
\hline Lectina & Insectos plaga & Actividad & Referencias \\
\hline PSA & Meligethes aeneus & Insecticida e insectistática & Melander et al., 2003 \\
ConA & Tarophagous proserpina & Insecticida & Powell, 2001 \\
Gleheda & Leptinotarsa decemlineata & Insectistática & Wang et al., 2003 \\
ConA & Callosobruchus maculatus & Insectistática & Sadeghi et al., 2006 \\
ConA & Helicoverpa armigera & Insectistática & Shukla et al., 2005 \\
GS-II & Callosobruchus maculatus & Insectistática & Zhu et al., 1996 \\
PHA & Callosobruchus maculatus & Insectistática & Gatehouse et al., 1984 \\
PHA-E & Empoasca fabae & Insecticida & Habibi et al., 1998 \\
\hline
\end{tabular}

Pisum sativum (PSA); Canavalia ensiformis (Concanavalina A (ConA)); Glechoma hederacea (Gleheda); GS-II: Griffonia simplicifolia aglutinina; Phaseolus vulgaris (PHA). Adaptado de G. Vandenborre et al., 2011. 
lectina afecta drásticamente el desarrollo de los insectos.

Otro efecto insectistático que se ha encontrado afecta la postura de huevos (Sadeghi et al., 2006). Lectinas con dominio de leguminosas como la ConA y PHA redujeron la oviposición de Callosobruchus maculatus. Esta disminución en el número de huevos fue dosis dependiente y no afectó el desarrollo o la emergencia del huevo (Sadeghi et al., 2006). Estos resultados sugieren que los efectos insectistáticos de las lectinas con dominio de leguminosa pueden variar si se combinan con otras lectinas con el mismo dominio, esto se puede deber a cambios en la especificidad de reconocimiento de los carbohidratos.

\section{Efecto insecticida}

Las lectinas con dominio de leguminosa también tienen efecto insecticida, como se observa en la Tabla 2; sin embargo, este efecto es marcadamente dependiente de la dosis y del tiempo del tratamiento. Por ejemplo, la lectina de Spenostylis stenocarpa al $5 \%(\mathrm{p} / \mathrm{p})$ mostró una mortalidad del 30 al $80 \%$ en larvas de gorgojo del frijol (C. maculatus) alimentadas con dietas artificiales (Machuka et al., 2000). Este efecto sobre la viabilidad contrasta con el anteriormente descrito, en el que dosis inferiores al $2 \%$ tienen un efecto sobre el desarrollo y tamaño de la larva (Machuka et al., 2000). Resultados similares se han obtenido con la ConA sobre la chicharra tara (Tarophagous proserpina) en donde se observó un $93 \%$ de mortalidad durante la ingestión de ConA (Powell, 2001). Adicionalmente, la lectina PSA (aglutinina de Pisum sativum) al 1\% reduce la supervivencia de las larvas del escarabajo Meligethes aeneus en un $84 \%$ después de cuatro días de los ensayos (Melander et al., 2003).

\section{MODO DE ACCIÓN DE LECTINAS CON DOMINIO DE LEGUMINOSAS}

El mecanismo preciso de acción de las lectinas vegetales sobre los insectos aún no se conoce completamente, pero hay evidencias que muestran que la unión de la lectina a moléculas glicosiladas en el intestino del insecto es un prerrequisito para la toxicidad (Van Damme, 2008; Michiels et al., 2010). Esta hipótesis se basa en la presencia de $\mathrm{N}$-glicanos en el intestino medio de los insectos (Fig. 4). Estos azúcares podrían interaccionar con algunas lectinas con dominio de leguminosas que se unen específicamente a manosa y a N-acetilglucosamina. Estas interacciones pueden ocurrir en la matriz peritrófica que es rica en manosa y en las células epiteliales del intestino medio (Michiels et al., 2010). Adicionalmente, se ha sugerido que lectinas como ConA que se une a manosa interactúan con receptores glicosilados en la superficie de las células del epitelio del estómago. Esta interacción afecta el metabolismo normal y la función de las

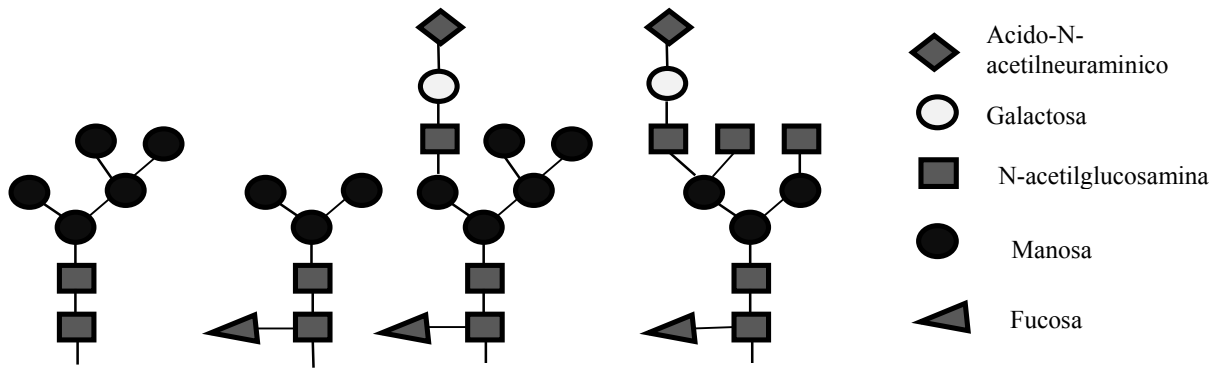

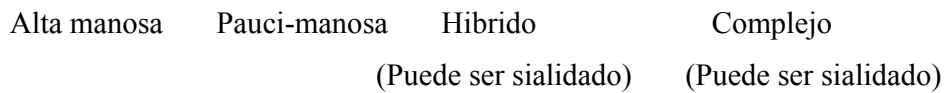

Fig. 4. Posibles estructuras de N-glicanos en insectos. Glicanos de insectos son de tipo altas manosas y pauci-manosas predominantemente, pero pequeñas cantidades de glicanos complejos han sido identificados. Glicanos con ácido siálico solo se han aislado de proteínas del sistema nervioso central.

Adaptado de: Michiels, K., E.J. Van Damme, and G. Smagghe. 2010. Archives of Insect Biochemistry and Physiology 73(4):193-212.

Fig. 4. Possible structures of N-glycans in insects. Insect glycans are high mannose and paucimannose type predominantly, but small amounts of complex glycans have been identified. Glycans with sialic acid only have been isolated from proteins of central nervous system.

Adapted from Michiels, K., E.J. Van Damme, and G. Smagghe. 2010. Archives of Insect Biochemistry and Physiology 73(4):193-212. 
células intestinales induciendo inflamación, hipersecreción y separación de la membrana apical, sin lisis y pérdida de la integridad de las células epiteliales, lo que resulta en una respuesta en la alimentación a largo plazo (Sauvion et al., 2004b). Además, se ha encontrado que las lectinas de varias plantas con especificidad hacia diferentes carbohidratos son tóxicas para las líneas celulares procedentes de tejidos de insectos lepidópteros (Shahidi-Noghabi et al., 2010)

El efecto crónico de las lectinas se atribuye a la resistencia a la proteólisis y a la estabilidad que tienen a un amplio rango de $\mathrm{pH}$ lo que garantiza una alta toxicidad oral (Vasconcelos y Oliveira, 2004; Michiels et al., 2010). Los insectos ingieren las lectinas a través de la alimentación y los primeros blancos para su unión son los receptores que podrían estar localizados en el tracto digestivo; sin embargo, si la proteína atraviesa la barrera del epitelio intestinal, un nuevo conjunto de receptores pueden ser el blanco de acción de las lectinas (Michiels et al., 2010).

Es importante recalcar que otras lectinas de unión a manosa pueden tener diferentes modos de acción a nivel celular o no presentar efecto, por lo que no se puede generalizar acerca de la acción de las mismas (Sauvion et al., 2004b). Este es el caso del estudio realizado por Habibi et al. (2000) en donde se determinaron los efectos de PHA en la estructura de células epiteliales del intestino medio del hemiptero Lygus hesperu. Los resultados obtenidos mostraron que la unión de la lectina a las microvellosidades del borde de cepillo generó una ruptura o ligero alargamiento de los núcleos. Por el contrario, otras isolectinas de PHA no tuvieron efecto en larvas del lepidóptero Lacanobia oleracea (Fitches et al., 2001). A pesar de que las dos isolectinas se unieron a glicopéptidos del intestino en los ensayos in vitro, no se encontró acumulación in vivo en los intestinos de los insectos (Fitches et al., 2001). Efectos similares se han encontrado en estudios con la lectina de Pisum sativum (PSA) que no tuvo efecto en la mortalidad, el peso y el tiempo de desarrollo de las larvas de abejas europeas (Apis mellifera) (Lehrman, 2007b). Estos resultados evidencian la selectividad de las lectinas y las diferencias en la forma de acción dependiendo del insecto (Abhilash et al., 2013).

Como se indicó en la sección anterior, el efecto insectistático y/o insecticida se da principalmente en los estados larvales y no en los estados adultos, y puede variar de insecto a insecto. Diferentes estudios han reportado estructuras complejas de glicanos en los insectos, con frecuencia en una sola etapa específica de su desarrollo (Tiemeyer et al., 2009). Por ejemplo, el ácido siálico se ha encontrado en embriones de la mosca de la fruta Drosophila melanogaster, pero no en la etapa adul- ta. Además, en las abejas pero no en otros insectos se ha reportado estructuras de glicanos que contiene un motivo GalNAc $\beta 1,4$ (Fuc $\alpha 1-3)$ GlcNAc $\beta 1-2$, lo que sugiere que los insectos pueden sintetizar estructuras complejas de glicanos de manera especie específicos (Tiemeyer et al., 2009). Wang et al. (2003) han propuesto que los efectos insecticidas de Gleheda están relacionados con su especificidad hacia el antígeno Tn $(\alpha-$ GalNAc unida a residuos Serina o Treonina de la cadena de polipéptidos), y que posiblemente en algunos insectos se expresa esta estructura en mucinas $u$ otras proteínas O-glicosiladas en el tracto digestivo; estas pueden actuar como receptores específicos para la unión de la lectina.

\section{PERSPECTIVAS Y CONCLUSIONES}

El mecanismo insectistático e insecticida de las lectinas con dominio de leguminosas no se ha dilucidado completamente. Sin embargo, hoy sabemos que afecta principalmente los estados larvales y que su efecto está mediado por la unión a carbohidratos como la manosa, N-acetilglucosamina y antígeno Tn que se encuentran en el intestino medio. Esta unión conlleva a una respuesta crónica que está mediada por la alta estabilidad de estas moléculas y que induce la inflamación, hipersecreción y separación de la membrana apical con o sin pérdida de la integridad celular de las células epiteliales del intestino medio. A pesar de que se ha dado un gran avance en la descripción de los efectos macros que tienen las lectinas con dominio de leguminosa, todavía hay un vacío respecto a las señales que se activan y que conllevan a estos efectos deletéreos. Esta información es necesaria para establecer modelos que permitan seleccionar con una alta eficacia el tipo de lectina que afecta un insecto plaga específico.

\section{LITERATURA CITADA}

Abhilash, J., K.V. Dileep, M. Palanimuthu, K. Geethanandan, C. Sadasivan, and M. Haridas. 2013. Metal ions in sugar binding, sugar specificity and structural stability of Spatholobus parviflorus seed lectin. J. Mol. Model. 19(8):3271-3278.

Al Atalah, B., G. Smagghe, and E.J. Van Damme. 2014. Orysata, a jacalin-related lectin from rice, could protect plants against biting-chewing and piercing-sucking insects. Plant Sci. 222:21-8.

Aldana, J.C. 2000. Estudio de la actividad y concentración de lectina en algunas subespecies de $S$. bogotensis y S. rubescens. Colombia, 2000. Tesis. Universidad Nacional de Colombia, Facultad de Ciencias, Bogotá, Colombia. 
Almanza, M., N. Vega, and G. Perez. 2004. Isolating and characterising a lectin from Galactia lindenii seeds that recognises blood group $\mathrm{H}$ determinants. Arch. Biochem. Biophys. 429(2):180-90.

Arcoverde, J.H., A.D. Carvalho, F.P. de Almeida Neves, B.P. Dionizio, E.V. Pontual, P.M. Paiva, et al. 2014. Screening of Caatinga plants as sources of lectins and trypsin inhibitors. Nat. Prod. Res. 28(16):1297-30.1.

Banerjee, R., K. Das, R. Ravishankar, K. Suguna, A. Surolia, and M. Vijayan. 1996. Conformation, protein-carbohydrate interactions and a novel subunit association in the refined structure of peanut lectin-lactose complex. J Mol Biol. 259(2):281-96.

Bezerra, M.J., N.V. Rodrigues, A.F. Pires, G.A. Bezerra, C.B. Nobre, K.L. Alencar, et al. 2013. Crystal structure of Dioclea violacea lectin and a comparative study of vasorelaxant properties with Dioclea rostrata lectin. The International Journal of Biochemistry \& Cell Biology 45(4):807-815.

Bhat, G.G., K.N. Shetty, N.N. Nagre, V.V. Neekhra, S. Lingaraju, R.S. Bhat, et al. 2010. Purification, characterization and molecular cloning of a monocot mannose-binding lectin from Remusatia vivipara with nematicidal activity. Glycoconj J 27(3):309-20.

Boulter, D., G.A. Edwards, A.M.R. Gatehouse, J.A. Gatehouse, and V.A. Hilder. 1990. Additive protective effects of different plant-derived insect resistance genes in transgenic tobacco plants. Crop Protection 9(5):351-354.

Bovi, M., M.E. Carrizo, S. Capaldi, M. Perduca, L.R. Chiarelli, M. Galliano, and H.L. Monaco. 2011. Structure of a lectin with antitumoral properties in king bolete (Boletus edulis) mushrooms. Glycobiology 21(8):1000-1009.

Castillo-Villanueva, A., H. Caballero-Ortega, F. Abdullaev-Jafarova, Y. Garfias, M. del Carmen Jimenez-Martinez, S. Bouquelet, et al. 2007. Lectin from Phaseolus acutifolius var. escumite: chemical characterization, sugar specificity, and effect on human T-lymphocytes. J. Agric. Food Chem. 55(14):5781-7.

Celis, Á., C. Mendoza, M. Pachón, J. Cardona, W. Delgado, y L.E. Cuca. 2008. Extractos vegetales utilizados como biocontroladores con énfasis en la familia Piperaceae. Una revisión. Agron. Colomb. 26(1):97-106.

Czapla, T.H., and B.A. Lang. 1990. Effect of plant lectins on the larval development of european corn borer (Lepidoptera: Pyralidae) and southern corn rootworm (Coleoptera: Chrysomelidae). J. Econ. Entomol. 83(6):2480-2485.
Chandra, N.R., M.M. Prabu, K. Suguna, and M. Vijayan. 2001. Structural similarity and functional diversity in proteins containing the legume lectin fold. Protein Engineering 14(11):857-866.

Chen, H., E. Gonzales-Vigil, C.G. Wilkerson, and G.A. Howe. 2007. Stability of plant defense proteins in the gut of insect herbivores. Plant Physiol. 143(4):1954-67.

Chen, M.S. 2008. Inducible direct plant defense against insect herbivores: A review. Insect Science 15(2):101-114.

Dam, T.K., S. Oscarson, R. Roy, S.K. Das, D. Page, F. Macaluso, and C.F. Brewer. 2005. Thermodynamic, kinetic, and electron microscopy studies of concanavalin A and Dioclea grandiflora lectin cross-linked with synthetic divalent carbohydrates. J. Biol. Chem. 280(10):8640-6.

Deacon, A., T. Gleichmann, A.J. Kalb, H. Price, J. Raftery, G. Bradbrook, et al. 1997. The structure of concanavalin A and its bound solvent determined with small-molecule accuracy at 0.94 ๑ resolution. Journal of the Chemical Society, Faraday Transactions 93(24):4305-4312.

De la Torre, P., B.A. Rocha, E.P. Souza, T.M. Oliveira, G.A. Bezerra, F.B. Moreno, et al. 2007. Structure of a lectin from Canavalia gladiata seeds: new structural insights for old molecules. BMC Struct. Biol. 7:52.

Fang, E.F., P. Lin, J.H. Wong, S.W. Tsao, and T.B. Ng. 2010. A lectin with anti-HIV-1 reverse transcriptase, antitumor, and nitric oxide inducing activities from seeds of Phaseolus vulgaris $\mathrm{cv}$. extralong autumn purple bean. J. Agric. Food Chem. 58(4):2221-9.

Fernández-Alonso, J.L., N. Vega, and G. Pérez. 2009. Lectin prospecting in Colombian Labiatae. A systematic-ecological approach- III. Mainly exotic species (cultivated or naturalised). Caldasia 31:227-245.

Fitches, E., C. Ilett, A. M.R. Gatehouse, L.N. Gatehouse, R. Greene, J.P. Edwards, and J.A. Gatehouse. 2001. The effects of Phaseolus vulgaris erythro- and leucoagglutinating isolectins (PHA-E and PHA-L) delivered via artificial diet and transgenic plants on the growth and development of tomato moth (Lacanobia oleracea) larvae; lectin binding to gut glycoproteins in vitro and in vivo. J. Insect Physiol. 47(12):1389-1398.

Gao, W., Y. Sun, S. Chen, J. Zhang, J. Kang, Y. Wang, H. Wang, G. Xia, Q. Liu, and Y. Kang. 2013. Mushroom lectin enhanced immunogenicity of HBV DNA vaccine in C57BL/6 and HBsAg-transgenic mice. Vaccine 31(18):227380. 
Gatehouse, F.M., Dewey, J. Dove, K.A. Fenton, and A. Pusztai. 1984. Effect of seed lectins from Phaseolus vulgaris on the development of larvae of Callosobruchus maculatus; mechanism of toxicity. Journal of the Science of Food and Agriculture 35(4):373-380.

Gatehouse, M.R., J.A. Gatehouse, and E. Fitches. 1997. Effects of snowdrop lectin (GNA) delivered via artificial diet and transgenic plants on the development of tomato moth (Lacanobia oleracea) larvae in laboratory and glasshouse trials. J. Insect Physiol. 43(8):727-739.

Goldstein, I.J., and C.E. Hayes. 1978. The lectins: carbohydrate-binding proteins of plants and animals. Adv. Carbohydr. Chem. Biochem. 35:127-340.

Goldstein, I.J., H.C. Winter, and R.D. Poretz. 1997. Plant lectins: tools for the studying of complex carbohydrates. p. 403-474. In Montreuil, J., Vliegenthart, J. f.G. and Schachter,H. (eds.) Glycoproteins II. Elsevier, Amsterdam, Netherland.

Goossens, A., W. Dillen, J. De Clercq, M. Van Montagu, and G. Angenon. 1999. The arcelin-5 Gene of Phaseolus vulgaris directs high seed-specific expression in transgenic Phaseolus acutifolius and Arabidopsis plants. Plant Physiology 120(4):1095-1104.

Habibi, J., E.A. Backus, and T.H Czapla. 1998. Subcellular effects and localization of binding sites of phytohemagglutinin in the potato leafhopper, Empoasca fabae (Insecta: Homoptera: Cicadellidae). Cell Tissue Res. 294(3):561-71

Habibi, J., E.A. Backus, and J.E. Huesing. 2000. Effects of phytohemagglutinin (PHA) on the structure of midgut epithelial cells and localization of its binding sites in western tarnished plant bug, Lygus hesperus Knight. J. Insect Physiol. 46(5):611-619.

Hamelryck, T.W., M.H. Dao-Thi, F. Poortmans, M.J. Chrispeels, L. Wyns, and R. Loris. 1996. The crystallographic structure of phytohemagglutinin-L. J. Biol. Chem. 271(34):20479-85.

Hamshou, M., E.J. Van Damme, S. Caccia, K. Cappelle, G. Vandenborre, B. Ghesquière, et al. 2013. High entomotoxicity and mechanism of the fungal GalNAc/Gal-specific Rhizoctonia solani lectin in pest insects. J. Insect Physiol. 59(3):295-305.

Hoffman, L.M., and D.D. Donaldson. 1985. Characterization of two Phaseolus vulgaris phytohemagglutinin genes closely linked on the chromosome. Embo. J. 4(4):883-9.

Jones, J.D., and J.L. Dangl. 2006. The plant immune system. Nature. 444 (7117): 323-9.
Kaneda, Y., R.F. Whittier, H. Yamanaka, E. Carredano, M. Gotoh, H. Sota, et al. 2002. The high specificities of Phaseolus vulgaris erythro- and leukoagglutinating lectins for bisecting GlcNAc or beta 1-6-linked branch structures, respectively, are attributable to loop B. J. Biol. Chem. 277(19):16928-16935.

Lam, S.K., Q.F. Han, and T.B. Ng. 2009. Isolation and characterization of a lectin with potentially exploitable activities from caper (Capparis spinosa) seeds. Biosci. Rep. 29(5):293-9.

Lam, S.K., and T.B. Ng. 2010. Isolation and characterization of a French bean hemagglutinin with antitumor, antifungal, and anti-HIV-1 reverse transcriptase activities and an exceptionally high yield. Phytomedicine 17(6):45762.

Lam, S.K., and T.B. Ng. 2011. Lectins: production and practical applications. Appl. Microbiol. Biotechnol. 89(1):45-55.

Lehrman, A. 2007b. Does pea lectin expressed transgenically in oilseed rape (Brassica napus) influence honey bee (Apis mellifera) larvae? Environmental Biosafety Research 6(04):271278.

Lehrman, A., I. Åhman, and B. Ekbom. 2007a. Influence of pea lectin expressed transgenically in oilseed rape (Brassica napus) on adult pollen beetle (Meligethes aeneus). Journal of Applied Entomology 131(5):319-325.

Leung, E.H., J.H. Wong, and T.B. Ng. 2008. Concurrent purification of two defense proteins from French bean seeds: a defensin-like antifungal peptide and a hemagglutinin. J. Pept. Sci. 14(3):349-53.

Loris, R. 2002. Principles of structures of animal and plant lectins. Biochimica et Biophysica Acta (BBA) - General Subjects 1572(2-3):198208.

Loris, R., T. Hamelryck, J. Bouckaert, and L. Wyns. 1998. Legume lectin structure. Biochimica et Biophysica Acta (BBA) - Protein Structure and Molecular Enzymology 1383(1):9-36.

Machuka, O.G. Okeola, M.J. Chrispeels, and L.E. Jackai. 2000. The African yam bean seed lectin affects the development of the cowpea weevil but does not affect the development of larvae of the legume pod borer. Phytochemistry 53(6):667-674.

Marrone, P.G. 2009. Barriers to adoption of biological control agents and biological pesticides. p. 163-178. In Edward B. Radcliffe, William D. Hutchison, and Rafael E. Cancelado (eds.) Integrated Pest Management. Cambridge University Press, Cambridge, UK. 
Melander, M., I. Ahman, I. Kamnert, and A.C. Stromdahl. 2003. Pea lectin expressed transgenically in oilseed rape reduces growth rate of pollen beetle larvae. Transgenic Res. 12(5):555-67.

Mello, M.O., and M.C. Silva-Filho. 2002. Plant-insect interactions: an evolutionary arms race between two distinct defense mechanisms. Brazilian Journal of Plant Physiology 14:7181.

Michiels, K., E.J. Van Damme, and G. Smagghe. 2010. Plant-insect interactions: what can we learn from plant lectins? Archives of Insect Biochemistry and Physiology 73(4):193-212.

Naeem, A., M. Saleemuddin, and R.H. Khan. 2007. Glycoprotein targeting and other applications of lectins in biotechnology. Curr. Protein Pept. Sci. 8(3):261-71.

Novakova, J., M. Ticha, and J. Kocourek. 1981. Studies on lectins. LI. The role of Mn2+ in the activity of the soybean lectin. Biochim. Biophys. Acta 670(3):401-7.

Pérez, G., and N. Vega. 2007. Lamiaceae lectins. Functional Plant Sci. Biotechnol. 1:288-299.

Pérez, G., N. Vega, and J.L. Fernández Alonso. 2006. Lectin prospecting in Colombian Labiatae. A systematic-ecological approach. II. Caldasia 28:179-196.

Permyakov, E. 2009. Metalloproteomics. John Wiley \& Sons, Inc, Hoboken, New Jersey, USA.

Perret, S., C. Sabin, C. Dumon, M. Pokorna, C. Gautier, O. Galanina, et al. 2005. Structural basis for the interaction between human milk oligosaccharides and the bacterial lectin PAIIL of Pseudomonas aeruginosa. Biochem. J. 389(Pt 2):325-32.

Powell, K.S. 2001. Antimetabolic effects of plant lectins towards nymphal stages of the planthoppers Tarophagous proserpina and Nilaparvata lugens. Entomologia Experimentalis et Applicata 99(1):71-78.

Pusztai, A., S.W. Ewen, G. Grant, W.J. Peumans, E.J. van Damme, L. Rubio, and S. Bardocz. 1990. Relationship between survival and binding of plant lectins during small intestinal passage and their effectiveness as growth factors. Digestion 2:308-16.

Rao, V.S., K. Lam, and P.K. Qasba. 1998. Architecture of the sugar binding sites in carbohydrate binding proteins: a computer modeling study. International Journal of Biological Macromolecules 23(4):295-307.

Sadeghi, A., E.J. Van Damme, W.J. Peumans, and G. Smagghe. 2006. Deterrent activity of plant lectins on cowpea weevil Callosobruchus maculatus (F.) oviposition. Phytochemistry 67(18):2078-84.
Sanz-Aparicio, J., J. Hermoso, T.B. Grangeiro, J.J. Calvete, and B.S. Cavada. 1997. The crystal structure of Canavalia brasiliensis lectin suggests a correlation between its quaternary conformation and its distinct biological properties from Concanavalin A. FEBS letters 405(1):114-118.

Sauvion, N., H. Charles, G. Febvay, and Y. Rahbé. 2004a. Effects of jackbean lectin (ConA) on the feeding behaviour and kinetics of intoxication of the pea aphid, Acyrthosiphon pisum. Entomologia Experimentalis et Applicata 110(1):31-44.

Sauvion, N., C. Nardon, G. Febvay, A.M. Gatehouse, and Y. Rahbe. 2004b. Binding of the insecticidal lectin Concanavalin $\mathrm{A}$ in pea aphid, Acyrthosiphon pisum (Harris) and induced effects on the structure of midgut epithelial cells. J. Insect Physiol. 50(12):1137-50.

Shahidi-Noghabi, S., E.J. Van Damme, M. Iga, and G. Smagghe. 2010. Exposure of insect midgut cells to Sambucus nigra L. agglutinins I and II causes cell death via caspase-dependent apoptosis. J. Insect Physiol. 56(9):11017.

Sharma, A., T.B. Ng, J.H. Wong, and P. Lin. 2009. Purification and characterization of a lectin from Phaseolus vulgaris cv. (Anasazi beans). J. Biomed. Biotechnol. Vol. 2009. Article ID 929568 doi:10.1155/2009/929568

Sharon. 2007. Lectins: Carbohydrate-specific reagents and biological recognition molecules. Journal of Biological Chemistry 282(5):27532764.

Sharon, N., and H. Lis. 1990. Legume lectins--a large family of homologous proteins. Faseb. J. 4(14):3198-208.

Sharon, N., and H. Lis. 2001. The structural basis for carbohydrate recognition by lectins. Adv. Exp. Med. Biol. 491:1-16.

Sharon, N., and H. Lis. 2004. History of lectins: from hemagglutinins to biological recognition molecules. Glycobiology 14(11):53R-62R.

Shukla, S., R. Arora, and H.C. Sharma. 2005. Biological activity of soybean trypsin inhibitor and plant lectins against cotton bollworm/legume pod borer, Helicoverpa armigera. Plant Biotechnology 22(1):1-6.

Singh, T., J.H. Wu, W.J. Peumans, P. Rouge, E.J. Van Damme, R.A. Alvarez, et al. 2006. Carbohydrate specificity of an insecticidal lectin isolated from the leaves of Glechoma hederacea (ground ivy) towards mammalian glycoconjugates. Biochem. J. 393(Pt 1):331-341. 
Srinivas, V.R., G.B. Reddy, N. Ahmad, C.P. Swaminathan, N. Mitra, and A. Surolia. 2001. Legume lectin family, the 'natural mutants of the quaternary state', provide insights into the relationship between protein stability and oligomerization. Biochim. Biophys Acta 15(3):102-111.

Tanji, T., A. Ohashi-Kobayashi, and S. Natori. 2006. Participation of a galactose-specific C-type lectin in Drosophila immunity. Biochem. J. 396(1):127-138.

Tiemeyer, M., S.B. Selleck, and J.D. Esko. 2009. Arthropoda. p. 347-362. In A. Varki, R. Cummings, J. Esko, H. Freeze, G. Hart, and J. Marth (eds.) Essentials of Glycobiology. 2nd ed. Cold Spring Harbor Laboratory Press, New York, USA.

Topin, J., J. Arnaud, A. Sarkar, A. Audfray, E. Gillon, S. Perez, et al. 2013. Deciphering the glycan preference of bacterial lectins by glycan array and molecular docking with validation by microcalorimetry and crystallography. PLoS One 8(8):e71149.

Upadhyay, S., and P. Singh. 2012. Receptors of garlic (Allium sativum) lectins and their role in insecticidal action. The Protein Journal 31(6):439-446.

Van Breedam, W., S. Pöhlmann, H.W. Favoreel, R.J. de Groot, and H.J. Nauwynck. 2013. Bitter-sweet symphony: glycan-lectin interactions in virus biology. FEMS Microbiology Reviews 38:598-632.

Van Damme, E.J. 2008. Plant lectins as part of the plant defense system against insects. 2nd ed. Springer Science, New York, USA.

Van Damme, E.J., L. Nausicaa, and W.J. Peumans. 2008. Plant lectins. Advances in Botanical Research 48:107-209.

Van Damme, E.J., W.J. Peumans, A. Barre, and P. Rougé. 1998a. Plant lectins: a composite of several distinct families of structurally and evolutionary related proteins with diverse biological roles. CRC Crit. Rev. Plant Sci. 17(6):575-692.

Van Damme, E.J., W.J. Peumans, A. Pusztai, and S. Bardocz. 1998b. Handbook of plant lectins: properties and biomedical applications. John Willey \& Sons, Chichester, UK.

Vandenborre, G., G. Smagghe, and E.J. Van Damme. 2011. Plant lectins as defense proteins against phytophagous insects. Phytochemistry 72(13):1538-50.

Varki, A., R. Cummings, J. Esko, H. Freeze, G. Hart, and J. Marth. 1999. Essentials of Glycobiology. Cold Spring Harbor, New York, USA.
Vasconcelos, I.M., and J.T. Oliveira. 2004. Antinutritional properties of plant lectins. Toxicon 44(4):385-403.

Vega, N., and G. Pérez. 2006. Isolation and characterisation of a Salvia bogotensis seed lectin specific for the Tn antigen. Phytochemistry 67(4):347-355.

Vijayan, M., and N. Chandra. 1999. Lectins. Current Opinion in Structural Biology 9(6):707714.

Wang, W., B. Hause, W.J. Peumans, G. Smagghe, A. Mackie, R. Fraser, and E.J. Van Damme. 2003. The Tn antigen-specific lectin from ground ivy is an insecticidal protein with an unusual physiology. Plant Physiology 132(3):1322-1334.

Wang, Y., Y. Liu, H. Wang, C. Li, P. Qi, and J. Bao. 2012. Agaricus bisporus lectins mediates islet $\beta$-cell proliferation through regulation of cell cycle proteins. Experimental Biology and Medicine 237(3):287-296.

Wecksler, M., A. Levy, and W.G. Jaffe. 1968. Mitogenic effects of extracts of Canavalia ensiformis and Con A. Acta Cient. Venez. 19(4):154-6.

Wei, X., J. Yang, X. Liu, D. Yang, J. Xu, J. Fang, and W. Wang. 2012. Identification and transcriptional analysis of two types of lectins (SgCTL-1 and SgGal-1) from mollusk Solen grandis. Fish Shellfish Immunol. 33(2):204-12.

Whetstone, P.A., and B.D. Hammock. 2007. Delivery methods for peptide and protein toxins in insect control. Toxicon 49(4):576-596.

Xia, L., and T.B. Ng. 2006. A hemagglutinin with mitogenic activity from dark red kidney beans. J. Chromatogr. B Analyt. Technol. Biomed. Life Sci. 844(2):213-6.

Yan, Q., Z. Jiang, S. Yang, W. Deng, and L. Han. 2005. A novel homodimeric lectin from Astragalus mongholicus with antifungal activity. Arch. Biochem. Biophys. 442(1):72-81.

Ye, X.Y., T.B. Ng, P.W. Tsang, and J. Wang. 2001. Isolation of a homodimeric lectin with antifungal and antiviral activities from red kidney bean (Phaseolus vulgaris) seeds. J. Protein Chem. 20(5):367-75.

Zhu, H., J. Du, K.M. Hui, P. Liu, J. Chen, Y. Xiu, et al. 2013. Diversity of lectins in Macrobrachium rosenbergii and their expression patterns under spiroplasma MR-1008 stimulation. Fish Shellfish Immunol. 35(2):300-9.

Zhu, K., J.E. Huesing, R.E. Shade, R.A. Bressan, P.M. Hasegawa, and L.L. Murdock. 1996. An insecticidal N-acetylglucosamine-specific lectin gene from Griffonia simplicifolia (Leguminosae). Plant Physiol. 110(1):195-202. 